\title{
Rapid monitoring the water extraction process of Radix Paeoniae Alba using near infrared spectroscopy
}

\author{
Tian $\mathrm{Hu}^{*, \S}$, Tongtong $\mathrm{Li}^{*, \S}$, Lei Nie*, Lixuan Zang*, Hengchang Zang*,* \\ and Yingzi Zeng ${ }^{\dagger}$ \\ *School of Pharmaceutical Sciences, Shandong University \\ Wenhuaxi Road 44, Jinan, 250012, P. R. China \\ ${ }^{\dagger}$ Shandong Wohua Pharmaceutical Technology Co., Ltd \\ Weifang, 261205, P. R. China \\ tzanghcw@126.com
}

Received 30 April 2016

Accepted 23 November 2016

Published 31 January 2017

\begin{abstract}
Near infrared (NIR) spectroscopy has been developed into one of the most important process analytical techniques (PAT) in a wide field of applications. The feasibility of NIR spectroscopy with partial least square regression (PLSR) to monitor the concentration of paeoniflorin, albiflorin, gallic acid, and benzoyl paeoniflorin during the water extraction process of Radix Paeoniae Alba was demonstrated and verified in this work. NIR spectra were collected in transmission mode and pretreated with smoothing and/or derivative, and then quantitative models were built up using PLSR. Interval partial least squares (iPLS) method was used for the selection of spectral variables. Determination coefficients $\left(R_{\text {cal }}^{2}\right.$ and $\left.R_{\text {pred }}^{2}\right)$, root mean squares error of prediction (RMSEP), root mean squares error of calibration (RMSEC), and residual predictive deviation (RPD) were applied to verify the performance of the models, and the corresponding values were 0.9873 and $0.9855,0.0487 \mathrm{mg} / \mathrm{mL}, 0.0545 \mathrm{mg} / \mathrm{mL}$ and 8.4 for paeoniflorin; 0.9879 , $0.9888,0.0303 \mathrm{mg} / \mathrm{mL}, 0.0321 \mathrm{mg} / \mathrm{mL}$ and 9.1 for albiflorin; $0.9696,0.9644,0.0140 \mathrm{mg} / \mathrm{mL}$, $0.0145 \mathrm{mg} / \mathrm{mL}$ and 5.1 for gallic acid; $0.9794,0.9781,0.00169 \mathrm{mg} / \mathrm{mL}, 0.00171 \mathrm{mg} / \mathrm{mL}$ and 6.9 for benzoyl paeoniflorin, respectively. The results turned out that this approach was very efficient and environmentally friendly for the quantitative monitoring of the water extraction process of Radix Paeoniae Alba.
\end{abstract}

Keywords: Near infrared spectroscopy; partial least squares regression; high performance liquid chromatography; Radix Paeoniae Alba.

\$Corresponding author.

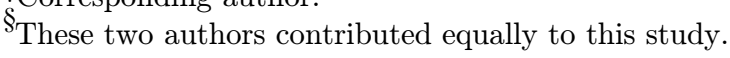

This is an Open Access article published by World Scientific Publishing Company. It is distributed under the terms of the Creative Commons Attribution 4.0 (CC-BY) License. Further distribution of this work is permitted, provided the original work is properly cited. 


\section{Introduction}

Radix Paeoniae Alba (Baishao), the dried root of Paeonia lactiflora Pall, one of the most widely used herb medicines has been reported to exhibit pharmacological actions such as anti-inflammatory, antiallergic, anti-thrombosis, immunoregulating, and kidney and liver protection properties. ${ }^{1-3}$ Monoterpene glucosides are generally considered to be the main bioactive compounds responsible for most of the biological activities. Among them, paeoniflorin is the major active constituent and has been used as a phytochemical marker for the quality control of Radix Paeoniae Alba in Chinese Pharmacopoeia. ${ }^{4}$ Other bioactive components, such as albiflorin, benzoyl paeoniflorin, gallic acid, etc., have also been reported as the pharmacologically active ingredients of Radix Paeoniae Alba. ${ }^{5-7}$

As one of the most popular raw herb materials for the production of Chinese patent medicines, such as Shenzhiling oral solution, $\mathrm{Si}-\mathrm{Wu}$ oral solution, Xiaoyaosan decoction, etc. Water extraction process is indispensible one of the key manufacturing units for most production of Chinese patent medicines containing Radix Paeoniae Alba. However, in large-scale traditional Chinese medicine (TCM) manufacturing process, the conventional extraction procedure is still the mainstream approach, and heat reflux extraction is the most widely used technique for the extraction of herbal materials. ${ }^{8}$ To improve the process efficiency and guarantee the final product quality, reliable process analytical technology (PAT) should be emphasized in the water extraction process of Radix Paeoniae Alba.

Near infrared (NIR) spectroscopy combined with chemometrics has found its useful applications in broad range of domains during the past decades, such as agricultural, food, pharmaceutical, and biomedical sectors. ${ }^{9-15}$ NIR spectroscopy has also shown great power and gained wide acceptance in TCM manufacturing industry. ${ }^{11,16}$ The most wellknown applications of NIR spectroscopy in the manufacturing processes of TCM include the separation monitoring, ${ }^{17}$ end point judgement of extraction process, ${ }^{18-20}$ alcohol precipitation monitoring, ${ }^{21}$ etc. Obviously, NIR spectroscopy is a promise technology for the understanding of the TCM manufacturing processes.

The objective of this study is to investigate the feasibility and application of NIR spectroscopy in determination of paeoniflorin, albiflorin, gallic acid, and benzoyl paeoniflorin during the water extraction process of Radix Paeoniae Alba. NIR calibration models were built up by using partial least squares regression (PLSR) with NIR spectra and reference data of samples collected from the water extraction process of Radix Paeoniae Alba. The performances of NIR calibration models were evaluated by means of determination coefficients $\left(R_{\text {cal }}^{2}\right.$ and $\left.R_{\text {pred }}^{2}\right)$, root mean squares error of prediction (RMSEP), root mean squares error of calibration (RMSEC), and residual predictive deviation (RPD). To our knowledge, this is the first report to demonstrate the feasibility of NIR spectroscopy with PLSR for rapid monitoring of the water extraction process of Radix Paeoniae Alba.

\section{Materials and Methods}

\subsection{Chemicals and reagents}

Authentic paeoniflorin, albiflorin, gallic acid, and benzoyl were obtained from National Institute for the Control of Pharmaceutical and Biological Products (Beijing, China). High performance liquid chromatography (HPLC) grade acetonitrile and methanol were purchased form Merck (Darmstadt, Germany). Radix Paeoniae Alba was provided by Wohua Pharmaceutical Technology Co., Ltd, (Weifang, China). Distilled water was obtained from Milli-Q water purification system of Millipore (Bedford, MA, USA). Other reagents were obtained from VWR International (South Plainfield, NJ, USA).

\subsection{Radix Paeoniae Alba water extraction and sampling}

Extraction of Radix Paeoniae Alba was carried out under laboratory-scale simulating the actual procedure of Radix Paeoniae Alba water extract. Thirty gram Radix Paeoniae Alba was soaked in $300 \mathrm{~mL}$ tap water at room temperature $\left(25^{\circ} \mathrm{C}\right)$ for $40 \mathrm{~min}$, and then refluxed for $150 \mathrm{~min}$ at the first extraction stage, after filtration of the extract, the residues were extracted by another $180 \mathrm{~mL}$ tap water for $100 \mathrm{~min}$ at the second extraction stage. Sampling was from the same bottleneck of the $500 \mathrm{~mL}$ three-necked round-bottomed flask by glue dropper and each sampling was $3 \mathrm{~mL}$. Samples were 
collected at the beginning of boiling during each extraction, and then every $15 \mathrm{~min}$ during the first extraction and $20 \mathrm{~min}$ during the second extraction, four extraction batches were carried out and 68 samples were collected, but one sample was spilled, and then 67 samples were left.

\subsection{Spectra acquisition}

NIR spectra were collected using an Antaris II Fourier transform NIR spectrophotometer (Themo Fisher, USA). Transmission spectra of samples were collected from $4000 \mathrm{~cm}^{-1}$ to $10,000 \mathrm{~cm}^{-1}$ at every $8 \mathrm{~cm}^{-1}$ path interval. Each spectrum was obtained by averaging 32 scans with the gain value of $4 \times$ (B screen). To avoid error from the outer environment, all samples were equilibrated to room temperature $\left(25^{\circ} \mathrm{C}\right)$ prior to NIR spectra collection. And the humidity was also kept at ambient level in the laboratory. Figure 1 shows the raw spectra of the extract samples.

\subsection{Determination of reference values}

The concentration of paeoniflorin, albiflorin, gallic acid, and benzoyl paeoniflorin in the extract samples were analyzed by HPLC coupled with UV detection (HPLC-UV). Chromatographic analysis was performed on an Agilent 1260 HPLC system (Agilent Corp., Santa Clara, CA, USA). An Agilent ZORBAX SB-C 18 column $(4.6 \times 250 \mathrm{~mm}$, $5 \mu \mathrm{m})$ was employed for chromatographic separation with a flow rate of $0.8 \mathrm{~mL} / \mathrm{min}$. The mobile phase was consisted of acetonitrile (mobile phase A) and $0.1 \%$ phosphate acid in distilled water (mobile phase B). Gradient elution was carried with the following profile: $0-5 \mathrm{~min}, 5 \% \mathrm{~A} ; 5-6 \mathrm{~min}, 5-15 \% \mathrm{~A}$; 20-21 min, $\quad 15-25 \% \mathrm{~A} ; \quad 30-31 \mathrm{~min}, \quad 25-35 \% \mathrm{~A}$;

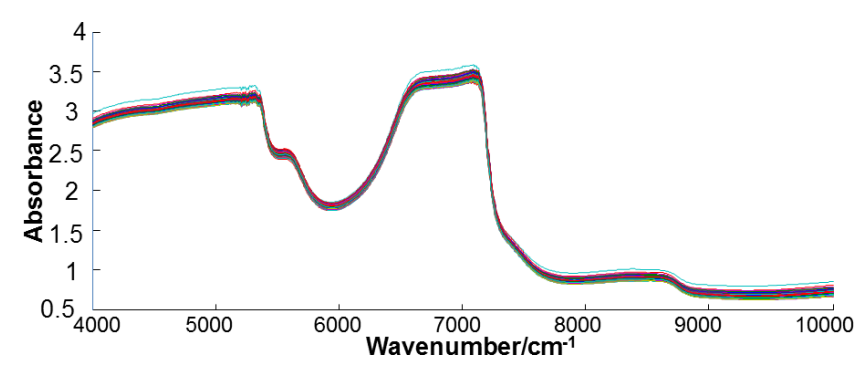

Fig. 1. Raw spectra of the extract samples in the water extraction process of Radix Paeoniae Alba.
31-42 min, $35-50 \%$; $42-45 \mathrm{~min}, \quad 50-5 \% \mathrm{~A}$; and $45-50 \mathrm{~min}, 5 \% \mathrm{~A}$. The wavelength of UV detector was set at $230 \mathrm{~nm}$ and the injection volume was $10 \mu \mathrm{L}$.

\subsection{Data analysis}

All the computations, including division of the calibration and validation set, spectral pretreatment, and variable selection were carried out using MATLAB version 2010a (MathWorks Inc., Natick, USA) with the PLS tool-box (version 752) purchased from Eigenvector Research, Inc., (Wenatchee, WA. USA). The NIR spectra and HPLC data were modeled by PLSR to establish the quantitative models.

\section{Results and Discussion}

\subsection{Reference values analysis}

The HPLC-UV method was developed and validated for the determination of the four analytes in the 67 extract samples. Chromatogram of one sample is shown in Fig. 2.

The main methodology parameters and calibration curves of the validated HPLC-UV method are listed in Table 1. The concentration of the four analytes in the extract samples are listed in Table 2. The range of the concentrations of gallic acid, albiflorin, paeoniflorin, and benzoyl paeoniflorin in the extract samples were $0.077-0.331,0.154-0.902$, $0.240-1.386$, and $0.011-0.044 \mathrm{mg} / \mathrm{mL}$, respectively.

\subsection{Division of calibration and validation set}

Firstly, the samples with the highest and lowest concentrations of gallic acid, albiflorin, paeoniflorin,

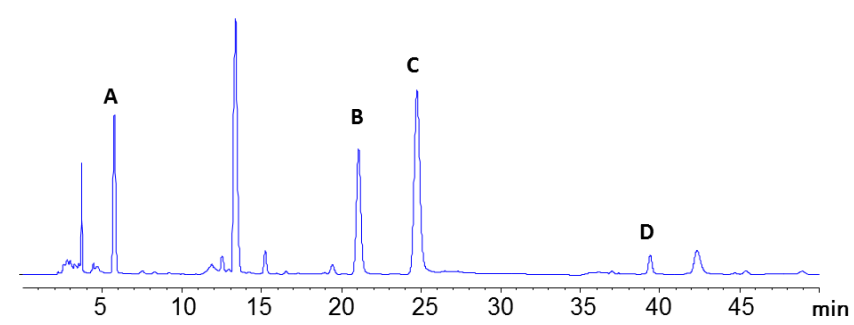

Fig. 2. Chromatogram of gallic acid (A), albiflorin (B), paeoniflorin (C) and benzoyl paeoniflorin (D). 
Table 1. Liner calibration functions and methodology parameters of HPLC-UV.

\begin{tabular}{|c|c|c|c|c|c|c|c|}
\hline \multirow[b]{2}{*}{ Analytes } & \multirow[b]{2}{*}{ Calibration curve } & \multirow[b]{2}{*}{$r^{2}$} & \multirow[b]{2}{*}{$\begin{array}{c}\text { Linear } \\
\text { range }(\mu \mathrm{g} / \mathrm{mL})\end{array}$} & \multicolumn{2}{|c|}{ Precision } & \multirow[b]{2}{*}{ Stability (RSD) } & \multirow[b]{2}{*}{$\begin{array}{c}\text { Repeatability } \\
\text { (RSD) }\end{array}$} \\
\hline & & & & $\begin{array}{l}\text { Intra-day } \\
\quad(\mathrm{RSD})\end{array}$ & $\begin{array}{l}\text { Inter-day } \\
(\mathrm{RSD})\end{array}$ & & \\
\hline Gallic acid & $y=0.0073 x-3.5131$ & 0.9995 & $3.0-60.0$ & $0.3 \%$ & $1.8 \%$ & $0.0 \%$ & $3.3 \%$ \\
\hline Albiflorin & $y=0.0132 x-0.2801$ & 0.9990 & $5.6-112.0$ & $0.0 \%$ & $1.1 \%$ & $0.9 \%$ & $2.9 \%$ \\
\hline Paeoniflorin & $y=0.0117 x-1.4902$ & 0.9994 & $6.9-138.6$ & $0.0 \%$ & $0.4 \%$ & $0.2 \%$ & $3.3 \%$ \\
\hline Benzoyl paeoniflorin & $y=0.0060 x-0.4921$ & 0.9978 & $0.7-6.5$ & $0.0 \%$ & $3.8 \%$ & $3.9 \%$ & $3.4 \%$ \\
\hline
\end{tabular}

Table 2. Results of the concentration determined by HPLC-UV.

\begin{tabular}{lcccc}
\hline & \multicolumn{4}{c}{ Concentration range $(\mathrm{mg} / \mathrm{mL})$} \\
\cline { 2 - 5 } Analytes & Batch 1 & Batch 2 & Batch 3 & Batch 4 \\
\hline Gallic acid & $0.105-0.331$ & $0.108-0.327$ & $0.077-0.310$ & $0.070-0.303$ \\
Albiflorin & $0.189-0.875$ & $0.236-0.902$ & $0.154-0.841$ & $0.142-0.797$ \\
Paeoniflorin & $0.292-1.349$ & $0.311-1.386$ & $0.254-1.326$ & $0.240-1.336$ \\
Benzoyl paeoniflorin & $0.014-0.040$ & $0.015-0.041$ & $0.013-0.041$ & $0.011-0.044$ \\
\hline
\end{tabular}

and benzoyl paeoniflorin were divided into the calibration set, while the remaining samples were split into two sets, calibration set and validation set, by Kennard-Stone (KS) algorithm. ${ }^{10-13}$ In current study, 53 samples ( $80 \%$ ) were placed in the calibration set, and the remaining 14 samples (20\%) were signed to the validation set. The ranges of the concentration of gallic acid, albiflorin, paeoniflorin, and benzoyl paeoniflorin in calibration and validation sets are listed in Table 3 and score plot of principle components (PCs) for sample division is shown in Fig. 3, obviously, samples belonging to the validation set are evenly distributed throughout the calibration set.

\subsection{Spectral pretreatments}

NIR spectral analysis has been widely applied in virtue of the development of chemometrics, in which spectral pretreatment and variable selection methods play an important role in the development of the robust NIR models. In our current study, different spectral pretreatment methods such as first derivative (FD) conversion, Savitzky-Golay (SG) filter, standard normal variate (SNV), and the combinations of them were used and compared. For the optimization of the spectral pretreatment methods, all of the variables $(n=1577)$ were included for the PLSR modeling. For each analyte, the optimized spectral preprocessing method was

Table 3. The concentration values of the four target components in the calibration and validation sets.

\begin{tabular}{llcc}
\hline Analytes & Sample set & Range $(\mathrm{mg} / \mathrm{mL})$ & Mean $(\mathrm{mg} / \mathrm{mL})$ \\
\hline Paeoniflorin & Calibration & $0.240-1.386$ & 0.950 \\
\multirow{3}{*}{ Albiflorin } & Validation & $0.311-1.374$ & 0.801 \\
& Calibration & $0.142-0.902$ & 0.597 \\
Gallic acid & Validation & $0.203-0.888$ & 0.504 \\
\multirow{3}{*}{ Benzoyl paeoniflorin } & Calibration & $0.070-0.331$ & 0.208 \\
& Validation & $0.106-0.305$ & 0.184 \\
& Calibration & $0.010-0.044$ & 0.0303 \\
\hline
\end{tabular}




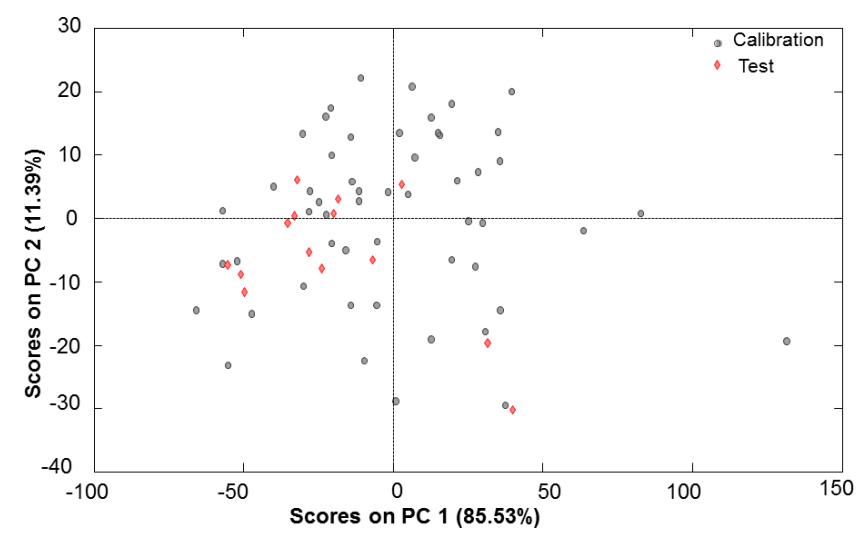

Fig. 3. PCA score plot of raw spectra for dividing sample sets. selected based on the RMSEC, RMSEP, $R_{\text {cal }}^{2}$, and $R_{\text {pred }}^{2}$ values of the PLSR model. Take the PLSR model of albiflorin for example, as shown in Table 4, the RMSEC, RMSEP, $R_{\text {cal }}^{2}$, and $R_{\text {pred }}^{2}$ value of the PLSR model based on the SNV spectra were $0.0415 \mathrm{mg} / \mathrm{mL}, 0.0530 \mathrm{mg} / \mathrm{mL}, 0.9772$, and 0.9709 , respectively, which was the best among all the processing methods we tested with lower RMSEC and RMSEP, and higher $R_{\text {cal }}^{2}$, and $R_{\text {pred }}^{2}$ values. The optimization of the spectral pretreatment methods for the PLSR models of gallic acid, paeoniflorin, and benzoyl paeoniflorin were carried out following the same rule, while the details were omitted here.

Table 4. Detailed comparison of albiflorin models with different spectral pretreatments.

\begin{tabular}{lccccc}
\hline Pretreatment & Spectrum range $\left(\mathrm{cm}^{-1}\right)$ & RMSEC $(\mathrm{mg} / \mathrm{mL})$ & $\mathrm{RMSEP}(\mathrm{mg} / \mathrm{mL})$ & $R_{\text {cal }}^{2}$ & $R_{\text {pred }}^{2}$ \\
\hline Raw & $4000-10,000$ & 0.0499 & 0.0565 & 0.9670 & 0.9655 \\
SG15+FD & $4000-10,000$ & 0.0774 & 0.0749 & 0.9206 & 0.9377 \\
SNV & $4000-10,000$ & 0.0415 & 0.0530 & 0.9772 & 0.9709 \\
SG15+FD, SNV & $4000-10,000$ & 0.0623 & 0.0682 & 0.9486 & 0.9479 \\
\hline
\end{tabular}

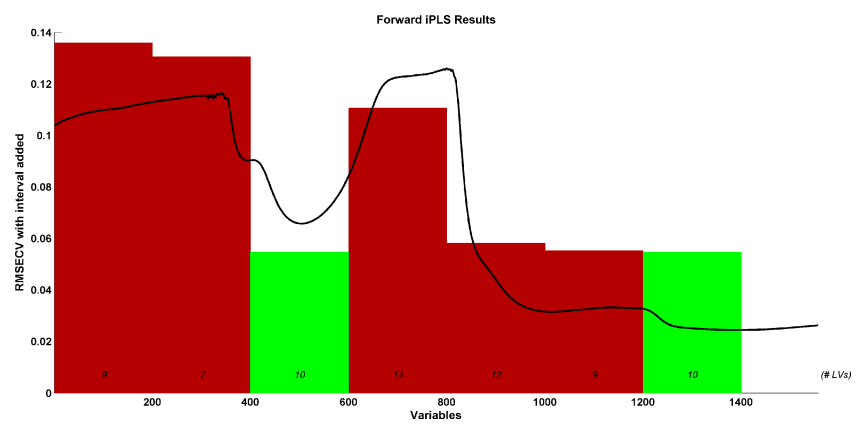

(a)

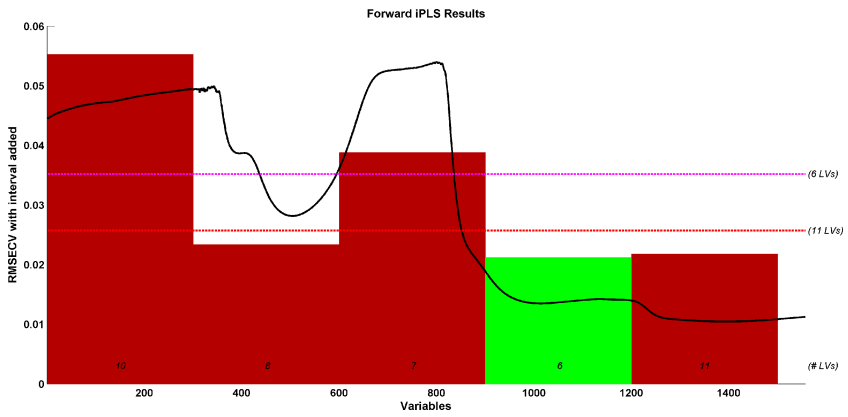

(c)

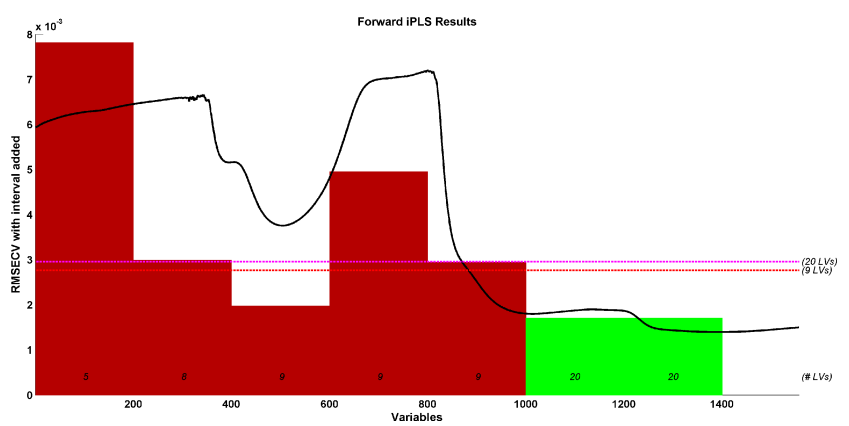

(b)

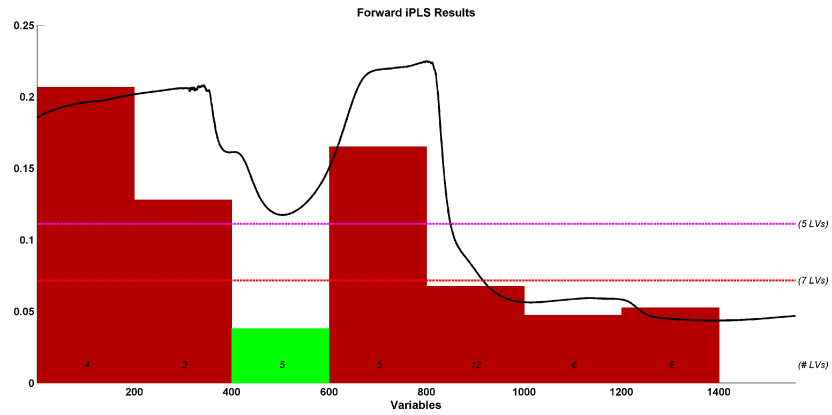

(d)

Fig. 4. The variable regions selected by forward iPLS for each PLSR model of the four analytes: (a) Paeoniflorin; (b) Benzoyl paeoniflorin; (c) Gallic acid; (d) Albiflorin. 


\subsection{Variable selection}

In this work, interval partial least square (iPLS) algorithm was first applied to split the full NIR spectral region (4000-10,000 $\mathrm{cm}^{-1}$ ) into different intervals; then, the optimized interval(s) was (were) achieved with the lowest RMSECV. Take the variable selection for the PLSR model of albiflorin as an example, the finally subinterval selected by iPLS is shown in Fig. 4(b) highlighted in green, corresponding to $5542-6310 \mathrm{~cm}^{-1}$. There were 200 variables selected for the modeling of albiflorin. Using the same approach, the interval(s) selected for gallic acid, paeoniflorin, and benzoyl paeoniflorin are shown in Figs. 4(a), 4(c) and 4(d), and the corresponding spectral regions are listed in Table 5. The spectral range for paeoniflorin model contains $5542.4-6309.9 \mathrm{~cm}^{-1}$ related to the first overtones of $-\mathrm{CH}_{3}$, and $-\mathrm{CH}_{2}-$ and $8627.9-9395.3 \mathrm{~cm}^{-1}$ related to the second $-\mathrm{OH}$ overtone. The spectral range for albiflorin model is $5542.4-6309.9 \mathrm{~cm}^{-1}$ related to the first overtones of $-\mathrm{CH}_{3}$, and $-\mathrm{CH}_{2}$ - and for gallic acid model is $7470.9-8624.0 \mathrm{~cm}^{-1}$ related to the second $\mathrm{C}-\mathrm{H}$ overtone and stretching and deformation combination. For benzoyl paeoniflorin model, the range of $7856.6-9395.3 \mathrm{~cm}^{-1}$ is

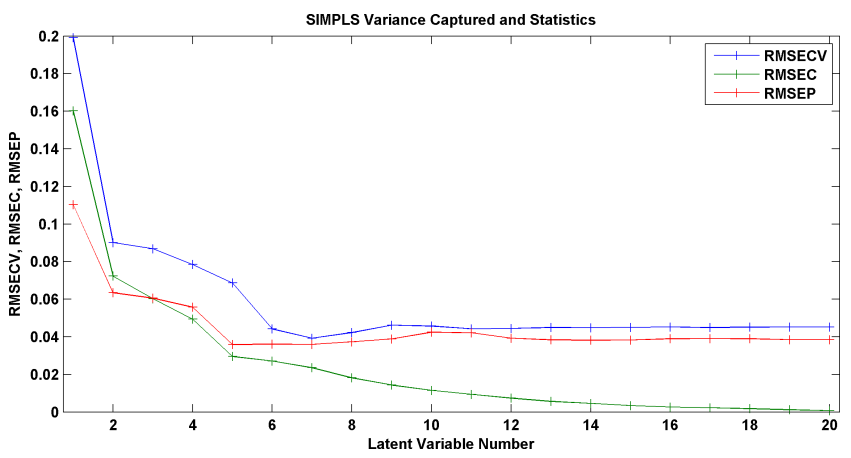

Fig. 5. RMSECV/RMSEC/RMSEP-LVs curves by "Venetian blinds" cross validation protocol of seven data splits for the PLSR model of albiflorin. associated with the second $-\mathrm{OH}$ overtone was selected.

Each calibration model has an optimal LVs number. LVs lower or greater than the optimal one introduced in the model may cause the problem of 'under-fitting' or 'over-fitting', both of which will lead to the decrease in predictability. In this research, take the PLSR model of albiflorin for example, the optimal number of LVs for this model was determined using a "Venetian blinds" cross validation protocol of seven data splits as shown in Fig. 5 . The model result with number 5 of LVs corresponds to the lower values of RMSEP, RMSEC and root mean square error of cross-validation (RMSECV), and the smaller difference between RMSEP and RMSEC, thus the number 5 of LVs was chosen as the optimal latent variable number for the albiflorin model. The same strategy was used to determine the most suitable LVs for the models of the other three analytes, and the results are listed in Table 5 .

\subsection{Evaluation of the calibration models}

The performance of the models was evaluated in terms of RMSEC, RMSEP, $R_{\text {cal }}^{2}$, and $R_{\text {pred }}^{2}$. To further evaluate the predictability of each PLS model, the standard deviation of the validation set to standard error of prediction ratio (RPD) was also calculated. A cutoff point of three was recommended by Williams and Sobering, ${ }^{22}$ and a higher value of RPD would be considered to have better predictive capability. Generally, a good model should have higher $R_{\text {cal }}^{2}, R_{\text {pred }}^{2}, \mathrm{RPD}$ and lower RMSEC, RMSEP, as well as small differences between RMSECV and RMSEP. Table 5 shows the performance indexes of the established models. The results shown in Fig. 6 indicate that the established models give satisfactory fitting results and predictive ability, and the respective models can be applied to monitor the concentrations of the four

Table 5. The performance indexes of each established model.

\begin{tabular}{lcccccccc}
\hline Analytes & Pretreatment & $\begin{array}{c}\text { Spectrum } \\
\text { range }\left(\mathrm{cm}^{-1}\right)\end{array}$ & LVs & $\begin{array}{c}\text { RMSEC } \\
(\mathrm{mg} / \mathrm{mL})\end{array}$ & $\begin{array}{c}\text { RMSEP } \\
(\mathrm{mg} / \mathrm{mL})\end{array}$ & $R_{\text {cal }}^{2}$ & $R_{\text {pred }}^{2}$ & RPD \\
\hline Paeoniflorin & SG15+FD & $5542.4-6309.9$ & 6 & 0.0487 & 0.0545 & 0.9873 & 0.9855 & 8.4 \\
& & $8627.9-9395.3$ & & & & & & \\
Albiflorin & SNV & $5542.4-6309.9$ & 5 & 0.0303 & 0.0321 & 0.9879 & 0.9888 & 9.1 \\
Gallic acid & Raw & $7470.9-8624.0$ & 6 & 0.0140 & 0.0145 & 0.9696 & 0.9644 & 5.1 \\
Benzoyl paeoniflorin & SNV & $7856.6-9395.3$ & 6 & 0.00169 & 0.00171 & 0.9794 & 0.9781 & 6.9 \\
\hline
\end{tabular}




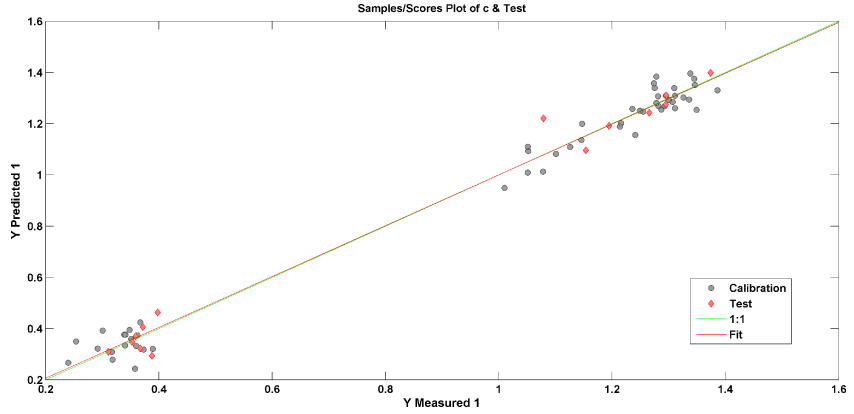

(a)

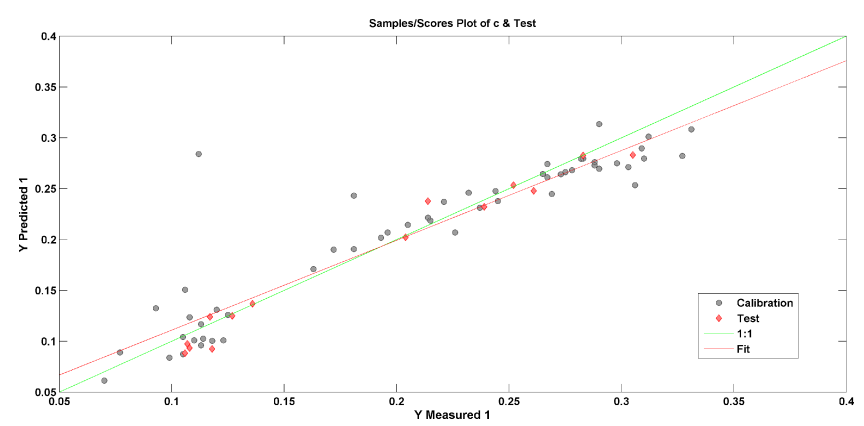

(c)

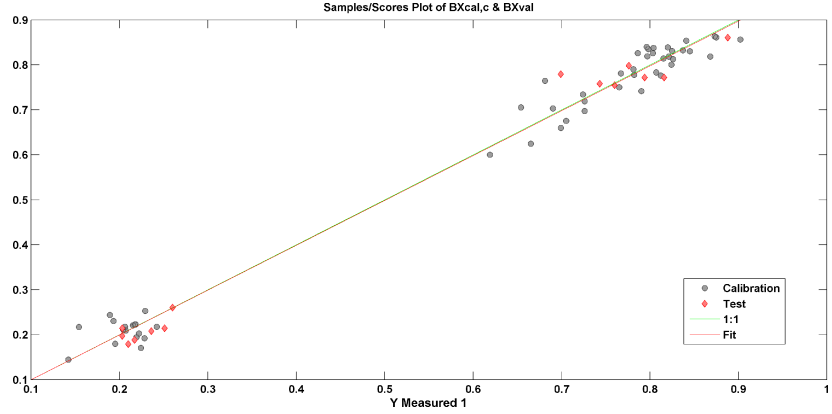

(b)

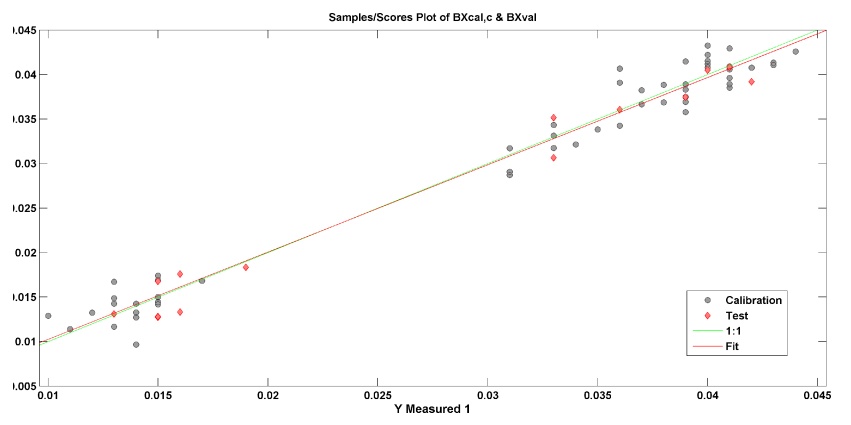

(d)

Fig. 6. The correlation charts of the four calibration models: (a) Paeoniflorin; (b) Albiflorin; (c) Gallic acid; (d) Benzoyl paeoniflorin.

analytes in the water extract during the water extraction process of Radix Paeoniae Alba.

\section{Conclusion}

In this study, a quantitative NIR spectroscopy method was explored and established for the simultaneous determination of paeoniflorin, albiflorin, gallic acid, and benzoyl paeoniflorin in the Radix Paeoniae Alba extract during the water extraction process of Radix Paeoniae Alba. By means of PLSR, quantitative NIR models were successfully built between the spectra and the corresponding reference values obtained by HPLC-UV. Compared with the HPLC-UV, the NIR spectroscopy method can significantly save manpower and time, and are potentially useful for monitoring the water extraction process of Radix Paeoniae Alba.

\section{Acknowledgments}

We are grateful for the financial support of the Basal Research Fund Project of Shandong University (No. 2015YQ010).

\section{References}

1. W. Li, S. Huang, R. Wang, "Advances in research on pharmacological actions and quality control of Paeoniae Radix Alba," Pharm. Care Res. 2, 016 (2012).

2. C. H. Wang, R. Wang, X. M. Cheng, Y. Q. He, Z. T. Wang, C. Wu, J. Cao, "Comparative pharmacokinetic study of paeoniflorin after oral administration of decoction of Radix Paeoniae Rubra and Radix Paeoniae Alba in rats," J. Ethnopharmacol. 117, 467-472 (2008).

3. H. Ohta, J. W. Ni, K. Matsumoto, H. Watanabe, M. Shimizu, "Peony and its major constituent, paeoniflorin, improved radial maze performance impaired by scopolamine in rats," Pharmacol. Biochem. Behav. 45, 719-723 (1993).

4. State Pharmacopoeia Committee of People's Republic of China, Chinese Pharmacopoeia, Chemical Industry Press, Beijing (2015).

5. K. S. Suh, E. M. Choi, Y. S. Lee, Y. S. Kim, "Protective effect of albiflorin against oxidativestress-mediated toxicity in osteoblast-like MC3T3E1 cells," Fitoterapia 89, 33-41 (2013).

6. T. Okubo, F. Nagai, T. Seto, K. Satoh, K. Ushiyama, I. Kano, "The inhibition of phenylhydroquinone-induced oxidative DNA cleavage by 
constituents of Moutan Cortex and Paeoniae Radix," Biol. Pharm. Bull. 23, 199-203 (2000).

7. G. C. Yen, P. D. Duh, H. L. Tsai, "Antioxidant and pro-oxidant properties of ascorbic acid and gallic acid," Food Chemistry 79, 307-313 (2002).

8. J. P. Fan, J. Cao, X. H. Zhang, J. Z. Huang, T. Kong, S. Tong, Z. Y. Tian, Y. L. Xie, R. Xu, J. H. Zhu, "Optimization of ionic liquid based ultrasonic assisted extraction of puerarin from Radix Puerariae Lobatae by response surface methodology," Food Chemistry 135, 2299-306 (2012).

9. Q. Dong, H. Zang, A. Liu, G. Yang, C. Sun, L. Sui, P. Wang, L. Li, "Determination of molecular weight of hyaluronic acid by near-infrared spectroscopy," $J$. Pharm. Biomed. Anal. 53, 274-278 (2010).

10. H. Huang, H. Yu, H. Xu, Y. Ying, "Near infrared spectroscopy for on/in-line monitoring of quality in foods and beverages: A review," J. Food Eng. 87, 303-313 (2008).

11. P. Wang, Z. Yu, "Species authentication and geographical origin discrimination of herbal medicines by near infrared spectroscopy: A review," J. Pharm. Anal. 5, 277-284 (2015).

12. E. W. Ciurczak, B. Igne, Pharmaceutical and Medical Applications of Near-Infrared Spectroscopy, CRC Press (2014).

13. A. Rohman, A. Nugroho, E. Lukitaningsih, Sudjadi, "Application of Vibrational Spectroscopy in Combination with Chemometrics Techniques for $\mathrm{Au}-$ thentication of Herbal Medicine," Appl. Spectrosc. Rev. 49, 603-613 (2014).

14. F. Wang, W. Jiang, C. Li, H. Zhang, L. Nie, L. Li, P. Wang, H. Zang, "Application of near infrared spectroscopy in monitoring the moisture content in freeze-drying process of human coagulation factor VIII," J. Innov. Opt. Health Sci. 8, 1550034 (2015).

15. P. Wang, J. Sun, T. Zhang, W. Liu, "Vibrational spectroscopic approaches for the quality evaluation and authentication of virgin olive oil," Appl. Spectrosc. Rev. 51, 763-790 (2016).

16. X. L. Chu, Y. P. Xu, W. Z. Lu, "Research and Application Progress of Chemometrics Methods in Near Infrared Spectroscopic Analysis," Chinese J. Anal. Chem. 5, 031 (2008).

17. H. Liu, X. Zhao, T. Qi, Y. P. Qi, G. R. Fan, "Establishment of the model for online monitoring of the column separation and purification process by near-infrared spectroscopy and determination of total ginsenosides in Folium Ginseng," Guang pu xue yu guang pu fen $x i=$ Guang pu 33, 3226-3230 (2013).

18. Y. Wu, Y. Jin, H. Ding, L. Luan, Y. Chen, X. Liu, "In-line monitoring of extraction process of scutellarein from Erigeron breviscapus (vant.) HandMazz based on qualitative and quantitative uses of near-infrared spectroscopy," Spectrochim. Acta A, Mol. Biomol. Spectrosc. 79, 934-939 (2011).

19. Z. Wu, C. Sui, B. Xu, L. Ai, Q. Ma, X. Shi, Y. Qiao, "Multivariate detection limits of on-line NIR model for extraction process of chlorogenic acid from Lonicera japonica," J. Pharm. Biomed. Anal. 77, 16-20 (2013).

20. P. Wang, H. Zhang, H. Yang, L. Nie, H. Zang, "Rapid determination of major bioactive isoflavonoid compounds during the extraction process of kudzu (Pueraria lobata) by near-infrared transmission spectroscopy," Spectrochim. Acta A, Mol. Biomol. Spectrosc. 137, 1403-1408 (2015).

21. Z. Wu, B. Xu, M. Du, C. Sui, X. Shi, Y. Qiao, "Validation of a NIR quantification method for the determination of chlorogenic acid in Lonicera japonica solution in ethanol precipitation process," J. Pharm. Biomed. Anal. 62, 1-6 (2012).

22. P. C. Williams, D. C. Sobering, How do we do it: A brief summary of the methods we use in developing near infrared calibrations, Near Infrared Spectroscopy: The Future Waves, 185-188 (1996). 